\title{
Dismetría Pélvica en una Muestra Radiológica de Adolescentes de la Ciudad de Temuco-Chile
}

\author{
Pelvic Asymmetry in a Radiographic Sample of Adolescents from the City of Temuco-Chile \\ "Héctor Silva M.; "Erika Collipal L.; ** Cristian Martínez F.; \\ ${ }^{* * *}$ Vanesa Carrasco A.; ${ }^{* * * *}$ Rene Rivas; ${ }^{* * * *}$ Enrique Montiel \& ${ }^{* * * * *}$ Tamara Silva F.
}

SILVA, M. H.; COLLIPAL, L. E.; MARTINEZ F. C.; CARRASCO, A. V.; RIVAS, R.; MONTIEL, E. \& SILVA, F. T. Dismetría pélvica en una muestra radiológica de adolescentes de la ciudad de Temuco-Chile. Int. J. Morphol., 30(1):94-99, 2012.

RESUMEN: La dismetría de la pelvis es frecuente en los adolescentes y puede ser un factor que provoque dolores en la columna vertebral y estar asociada a escoliosis funcionales de la región lumbar. Estas disfunciones de la columna vertebral producen incapacidad laboral, son de alta prevalencia en el mundo. Este problema de salud pública es de origen multifactorial afecta a la mayoría de las personas principalmente en la edad media de la vida. El propósito del estudio fue conocer cómo se comportan nuestros adolescentes con respecto a la variable dismetría pélvica. Para ello se analizaron 49 radiografías panorámicas de columna vertebral, de escolares entre 13 y 17 años de ambos géneros, 24 hombres y 25 mujeres, de la ciudad de Temuco, Chile. Del total de sujetos, 33 presentaron algún grado de desnivel pélvico lo que corresponde al 67,3\% de los individuos. En 27 sujetos estaban en el rango 1 a 10 mm y 4 sujetos en el rango de 11 a 15 mm. En 33 sujetos tenían algún grado de desviación de la columna lumbar en el plano frontal y 16 sujetos no se observó desviación. Es importante conocer estos parámetros en nuestra población adolescente, para incorporar a la educación formal la prevención en todos los aspectos de la vida y así disminuir la sintomatología, costos de atención y rehabilitación.

PALABRAS CLAVE: Pelvis; Desnivel pélvico; Columna lumbar.

\section{INTRODUCCIÓN}

El dolor lumbar es la segunda causa de discapacidad en los adultos y en Estados Unidos de Norteamérica (EEUU) existen pérdidas de trabajo asociados a dolores del sistema músculo-esquelético y, de dinero, que se estiman en aproximadamente 61,7 mil millones de dólares por año (Mackenzie, 1998; Centers for Disease Control and Prevention, 2001; Stewart et al., 2003).

Frimoyer et al. (1983) comunicaron una prevalencia del dolor de espalda a lo largo de la vida del $70 \%$, parecida a la comunicada por autores daneses, suecos y holandeses. En Canadá, la incidencia anual de lumbalgia causante de ausentismo laboral fue de 1,4\%, en 1981. En Inglaterra se han comunicado tasas de $4,5 \%$ en estudios transversales que cubrían un gran margen de oficios (Anderson, 1986).

El dolor región inferior de la espalda (DRIE) suele empezar en etapas precoces de la vida; en los escolares es más frecuente de lo que antes se pensaba, pero suele ser ligero y de corta duración. Balague et al. (1988) estudiaron 1.715 escolares en una comunidad Suiza, de los cuáles un $35 \%$ tenía antecedentes de DRIE y su prevalencia de una semana era del $16 \%$. Hasta ese momento, un $14 \%$ había buscado consejo médico. Como la causa del dolor de espalda en los niños puede ser un proceso grave, los pocos niños que manifiestan dolor importante requerían una investigación diagnóstica cuidadosa. En estos momentos la obesidad también es un factor que produce disminución en la funcionalidad de la columna, con aumento de las curvaturas y anteversion pélvica (Vismara et al., 2010).

Por la alta frecuencia de dolores de la columna vertebral, habitualmente se detectan en controles de salud de los adolescentes dismetría en los miembros inferiores, pelvis, columna vertebral, y estas diferencias son normales, como lo evidencian los estudios hechos en el mundo, en Alemania y EEUU, se detectó un $68 \%$ de dismetría, por lo tanto, al parecer los dos tercios de la población presentan

\footnotetext{
* Departamento de Ciencias Básicas. Universidad de La Frontera, Temuco, Chile.

** Departamento de Educación Física. Universidad de La Frontera, Temuco, Chile.

*** Facultad de Ciencias de la Salud. Universidad Autónoma de Chile, Temuco, Chile.

***** BIOREN-CEBIOR. Departamento de Ciencias Básicas. Universidad de La Frontera, Temuco, Chile.

*****Ayudante de Investigación. Carrera de Biotecnología. Universidad de La Frontera, Temuco, Chile.
} 
cierto grado de dismetría y desequilibrios. Florenciano \& Gironés (2009), esto también afecta al sistema craneomandibular y viceversa (Cuccia \& Caradonna, 2009).

En Chile, Fuentes et al. (1999) en su estudio sobre influencia de la postura corporal en la prevalencia de las disfunciones craneomandibulares (DCM) en radiografías, manifiesta que algunos síntomas de DCM son más frecuentes cuando existe una falta de alineación de las caderas y de los hombros, lo cual se observa principalmente en la sensibilidad muscular, pero no logro determinar que los síntomas de DCM se asociaban a una cadera más baja.

Silva (2001), en el trabajo sobre ángulo del plano sagital de la columna vertebral en radiografías, encontró que el $44 \%$ de la muestra era hiperlordótica y que presentaban altos niveles de estrés en la articulación lumbosacra.

Del Sol \& Hunter (2004) llevaron a cabo una evaluación postural en una muestra de la etnia mapuche entre 3 y 70 años, de la zona costera de la IX Región años encontrando que las alteraciones posturales observadas en el plano frontal se concentran a nivel de la columna vertebral, pelvis, rodilla y pies, también se observó en la silueta lateral que un $44 \%$ presentaba anteversión de la pelvis.

Espinoza-Navarro et al. (2009) en la ciudad de Arica, estudio una muestra de 120 alumnos, de 4 años de edad, encontrando que la mayor alteración era la inclinación de hombro con un $86 \%$.
Penha et al. (2005) encontró en su estudio de evaluación de la postura en niñas entre 7 y 10 años, variadas alteraciones tales como escoliosis, hiperlordosis lumbar, inclinación lateral de la pelvis (70\% de la muestra), hiperextensión de rodilla y rotación del tronco, hipercifosis, antepulsión.

Algunos autores sugieren que la inclinación de la pelvis es un factor importante en la aparición de la escoliosis lumbar no progresiva debido a desigualdad en la longitud de las piernas, lo que representa casi el $40 \%$ de las curvas por esta razón se ha asociado que la inclinación trae como compensación una escoliosis lumbar, que podría tener como causa primaria una desigualdad aparente o real de longitud de los miembros inferiores, que podrían generar dolores lumbares (Morote 1976; Dickson et al., 1983; Cummings et al., 1993; Gross et al., 1998).

Según las investigaciones clínicas, las mediciones radiografías tienen un nivel de confianza mayor que las marcas anatómicas sobre la piel (Beal, 1950; Clark, 1972; Friberg et al., 1985; Fann et al., 1999).

Estos datos epidemiológicos y clínicos han sido el motivo para efectuar un estudio radiológico de nuestra población adolescente, ya que se desconocen los parámetros ante enunciados en la Región de la Araucanía, Chile. Desde este punto de vista, es necesario tener un conocimiento de la morfología de la pelvis y columna vertebral de este grupo etario y de las variables que son potencialmente cambiantes a través del tiempo, debido a alteraciones en la postura, tonicidad muscular, enfermedades, etc.

\section{MATERIAL Y METODO}

El estudio se realizó en 49 adolescentes, de edades entre 13 y 17 años, de ambos sexos, alumnos de $8^{\circ}$ año básico de escuelas municipalizadas de la IX Región, que estaban conectadas a la Red Enlace de Informática Educativa. La toma de radiografías se llevó a cabo con el individuo sin ropa, de pie, descalzo, las dos rodillas completamente extendidas y paralelas. Se tomaron en la mañana radiografías panorámicas de la columna vertebral desde los planos sagital y frontal. La muestra fue tomada al azar, realizándose un muestreo aleatorio sistemático, en el cuál se determinó el espaciamiento de muestreo, la unidad de arranque fue de 4 para un universo de 2000 sujetos. Para la medición del desnivel pélvico se usó el método descrito por Tilley (1966) e Irvin (1991) (Fig. 1). Los datos fueron procesados con el programa estadístico SPSS 17.0. La dismetría fue medida por medio de un calíper digital;
Fig. 1. Radiografía que muestra los puntos que evalúan el desnivel pélvico. Línea A representa a una línea paralela a la línea de eburnación. Las líneas B, son líneas paralelas a la plomada a través de las cabezas femorales. La línea C es una perpendicular a la línea de la plomada. D es el valor de la dismetría. Tilley, P.; 1966.; Irvin, R. 1991. 
en un negatoscopio. Además, las radiografías fueron evaluadas e informadas por un médico radiólogo. Para el procesamiento de los datos se usó el programa estadístico SPSS versión 17.0 para Windows, analizando los estadísticos descriptivos e inferencial.

\section{RESULTADOS}

De acuerdo a lo resultados existe aproximadamente un $30,6 \%$ de individuos sin desnivel, y un $53 \%$ de los individuos con desnivel entre los 1 y $10 \mathrm{~mm}$. El resto de los sujetos un 16,4\% en encuentran en los rangos de 11-20 mm. Los principales resultados del estudio se observan en las Tablas I, II, III y IV.

\section{DISCUSIÓN}

Existen factores que contribuyen a la estabilidad de la postura, ya sea mecánicos o dinámicos, cualquier elemento que tenga una defunción puede acarrear compensaciones para mantener la postura y minimizar el gasto energético, de este punto de vista, este equilibrio se basa entre otra cosas en la visión horizontal, situando los dos ojos en forma paralela al plano del horizonte, lo que exige al sistema nervioso, músculos, huesos, articulaciones trabajar en conjunto.

Los factores que influencian la mala postura pueden ser intrínsecos o intrínsecos, tales como la herencia, el ambiente, condición física en cuales el sujeto vive, nivel

Tabla I. Valores de dismetría pélvica en relación al género en una muestra de radiografías de la pelvis de adolescentes de la ciudad de Temuco-Chile. No existe diferencias estadísticamente significativas entre los géneros, en relación a la diferencia de promedios del desnivel. $\mathrm{p}=0,4$.

\begin{tabular}{lcccccc}
\hline Desnivel $(\mathbf{m m})^{*}$ & \multicolumn{2}{c}{ Masculino } & \multicolumn{2}{c}{ Femenino } & \multicolumn{2}{c}{ Total } \\
\cline { 2 - 7 } & $\mathbf{n}$ & $\boldsymbol{\%}$ & $\mathbf{n}$ & $\boldsymbol{\%}$ & $\mathbf{n}$ & $\boldsymbol{\%}$ \\
\hline Sin desnivel & 9 & 18,4 & 6 & 12,2 & 15 & 30,6 \\
$1-4 \mathrm{~mm}$ & 4 & 8,2 & 10 & 20,4 & 14 & 28,5 \\
$5-10 \mathrm{~mm}$ & 6 & 12,2 & 6 & 12,2 & 12 & 24,5 \\
$11-15 \mathrm{~mm}$ & 3 & 6,1 & 3 & 6,2 & 6 & 12,3 \\
$16-20 \mathrm{~mm}$ & 1 & 2,0 & 1 & 2,1 & 2 & 4,1 \\
Total & 23 & 46,9 & 26 & 53,1 & 49 & 100 \\
\hline
\end{tabular}

Tabla II. Dismetría pélvica, asociado a la edad y lado de desviación convexa de la columna lumbar en una muestra de radiografías de la pelvis de adolescentes de la ciudad de Temuco-Chile.

\begin{tabular}{|c|c|c|c|c|c|c|c|c|}
\hline \multirow[t]{3}{*}{ Sexo/desnivel } & \multicolumn{8}{|c|}{ Lado convexidad de la columna lumbar } \\
\hline & \multicolumn{2}{|c|}{ Derecha } & \multicolumn{2}{|c|}{ Izquierda } & \multicolumn{2}{|c|}{ Normal } & \multicolumn{2}{|c|}{ Total } \\
\hline & $\mathbf{n}$ & $\%$ & $\mathbf{n}$ & $\%$ & $\mathbf{n}$ & $\%$ & $\mathbf{n}$ & $\%$ \\
\hline \multicolumn{9}{|l|}{ Masculino } \\
\hline Sin desnivel & 4 & 8,2 & 2 & 4,1 & 3 & 6,1 & 9 & 18,4 \\
\hline $1-4 \mathrm{~mm}$ & 2 & 4,1 & & & 2 & 4,1 & 4 & 8,1 \\
\hline $5-10 \mathrm{~mm}$ & 2 & 4,1 & 1 & 2,1 & 3 & 6,1 & 6 & 12,2 \\
\hline $11-15 \mathrm{~mm}$ & 1 & 2,1 & 1 & 2,1 & 1 & 2,1 & 3 & 6,1 \\
\hline 16 y más & 1 & 2,1 & & & & & 1 & 2,1 \\
\hline \multicolumn{9}{|l|}{ Femenino } \\
\hline Sin desnivel & 2 & 4,1 & 2 & 2,1 & 2 & 4,1 & 6 & 12,2 \\
\hline $1-4 \mathrm{~mm}$ & 2 & 4,1 & 5 & 10,2 & 3 & 6,1 & 10 & 20,4 \\
\hline $5-10 \mathrm{~mm}$ & 2 & 4,1 & 3 & 6,1 & 1 & 2,1 & 6 & 12,2 \\
\hline $11-15 \mathrm{~mm}$ & 1 & 2,1 & 1 & 2,1 & 1 & 2,1 & 3 & 6,1 \\
\hline 16 y más & 1 & 2,1 & & & & & 1 & 2,1 \\
\hline Total & 18 & 36,7 & 15 & 30,7 & 16 & 32,6 & 49 & 100 \\
\hline
\end{tabular}


SILVA, M. H.; COLLIPAL, L. E.; MARTINEZ F. C.; CARRASCO, A. V.; RIVAS, R.; MONTIEL, E. \& SILVA, F. T. Dismetría pélvica en una muestra radiológica de adolescentes de la ciudad de Temuco-Chile. Int. J. Morphol., 30(1):94-99, 2012.

Tabla III. Valor de la dismetría pélvica y lado del cuerpo con desnivel en relación al sexo en una muestra de radiografías de adolescentes de la ciudad de Temuco-Chile.

\begin{tabular}{|c|c|c|c|c|c|c|c|c|}
\hline \multirow[t]{2}{*}{ Sexo/desnivel } & \multicolumn{2}{|c|}{ Igual nivel } & \multicolumn{2}{|c|}{ Lado Derecho más bajo } & \multicolumn{2}{|c|}{ Lado Izquierdo más bajo } & \multicolumn{2}{|c|}{ Total } \\
\hline & $\mathbf{n}$ & $\%$ & $\mathbf{n}$ & $\%$ & $\mathbf{n}$ & $\%$ & $\mathbf{n}$ & $\%$ \\
\hline \multicolumn{9}{|l|}{ Masculino } \\
\hline Sin desnivel & 9 & 18,4 & & & & 18,4 & 9 & \\
\hline $1-4 \mathrm{~mm}$ & 1 & 2,1 & 1 & 2,1 & 2 & 4,1 & 4 & 8,2 \\
\hline $5-10 \mathrm{~mm}$ & & & 2 & 4,1 & 4 & 8,2 & 6 & 18,4 \\
\hline $11-15 \mathrm{~mm}$ & & & 3 & 6,1 & & & 3 & 6,1 \\
\hline 16 y más & & & 1 & 2,1 & & & 1 & 2,1 \\
\hline \multicolumn{9}{|l|}{ Femenino } \\
\hline Sin desnivel & 6 & 18,4 & & & & & 6 & 18,4 \\
\hline $1-4 \mathrm{~mm}$ & & & 7 & 14,3 & 3 & 6,1 & 10 & \\
\hline $5-10 \mathrm{~mm}$ & & & 5 & 10,1 & 1 & 2,1 & 6 & 18,4 \\
\hline $11-15 \mathrm{~mm}$ & & & 3 & 6,1 & & & 3 & 6,1 \\
\hline 16 y más & & & 1 & 2,1 & & & 1 & 2,1 \\
\hline Total & 16 & 32,7 & 15 & 46,9 & 10 & 20,4 & 49 & 100 \\
\hline
\end{tabular}

Tabla IV. Valores de la estadística descriptiva y de la prueba T pareada y R Pearson de la dismetría pélvica en una muestra de radiografías de adolescentes de la ciudad de Temuco, Chile.

\begin{tabular}{|c|c|c|c|}
\hline Estadística descriptiva e inferencial & Valores (mm) & Significancia & Correlación r Pearson \\
\hline Promedio (Hombres y Mujeres) & 4,6 & 0,4 & 0,55 \\
\hline Mediana & 4,0 & & \\
\hline Moda & 0,0 & & \\
\hline Máximo & 17,0 & & \\
\hline Mínimo & 0,0 & & \\
\hline
\end{tabular}

socioeconómico, factores emocionales y alteraciones fisiológicas debido al crecimiento y desarrollo.

La adolescencia es un periodo con cambios, los cuales se producen adaptaciones y ajuste en el cuerpo, estos ajustes pueden producir alteraciones que producen desbalances musculares que pueden generar dolores de espalda que afectan a gran proporción de la población siendo su prevalencia de aproximadamente un 70 a $80 \%$ y su incidencia de 3 a 5\%, según Carey et al. (1995) y McKinnon et al. (1997).

Estos dolores muchas veces van acompañado de alteraciones posturales que son a menudo encontrados en los niños y adolescentes (Del Sol \& Hunter; Silva; EspinozaNavarro et al.) que producen tensión en las estructuras de soporte.

La asimetría de la pelvis es común (Levangie, 1999; Krawiec et al., 2003) y por esta razón se especula también que podrían manifestarse síntomas neurológicos que provocan tensión en la meninge, cordón espinal, y tronco encefálico (Timgren \& Soinila, 2006).

El desnivel más allá de $5 \mathrm{~mm}$ puede causar síntomas dolorosos, pero menos de $5 \mathrm{~mm}$ podría llevar a compensaciones a nivel de la columna y pelvis tales como inclinaciones laterales, rotaciones de la pelvis. Nuestros adolescentes mostraron una alta prevalencia de dismetría con $67,3 \%$ de la muestra, con un valor promedio de 4,6 mm, que varió de 1 a 17 mm, encontrándose 26 (55\%) sujetos entre los rangos de 1-10 mm que está en concordancia con Penha et al.

En relación a la curvatura de la columna lumbar en la muestra, esta se desplaza hacia la derecha e izquierda en aproximadamente 34 sujetos $(69,4 \%)$, y $15(30,6 \%)$, siendo esta diferencia estadísticamente significativa, $(\mathrm{p}=0,000)$ en otra palabras la existencia del desnivel es compatible con una alteración a nivel de la columna lumbar en el plano 
frontal, pero sin ser una verdadera escoliosis, si no una compensación a la existencia del desnivel dicho por Penha et al., Dickson et al. y Gross et al.

Se ha asociado este signo de dismetría pélvica a dolores de la columna lumbar, con discrepancia de la longitud de las piernas, que se manifiesta en la adolescencia y en el adulto joven, con dolores recurrentes o crónicos, pero también este acortamiento o discrepancia puede afectar los hombros, tórax, rodillas y pie (Beal; Okun et al., 1982; Gofton \& Trueman, 1971; Blake \& Ferguson, 1992).
Finalmente, creemos que es necesario implementar pautas de prevención en el ámbito primario, por ejemplo al nivel de los establecimientos de enseñanza media, como parte del currículo de los estudiantes. Esta forma de prevención ayudaría a disminuir en parte la aparición de esta sintomatología, de los gastos en tratamientos y rehabilitación.

AGRADECIMIENTOS. Los autores agradecen el excelente apoyo profesional al Dr. Marabolit durante esta investigación.

SILVA, M. H.; COLliPAL, L. E.; MARTINEZ F. C.; CARRASCO, A. V.; RIVAS, R.; MONTIEL, E. \& SILVA, F. T. Pelvic asymmetry in a radiographic sample of adolescents from the city of Temuco-Chile. Int. J. Morphol., 30(1):94-99, 2012.

SUMMARY: Lower limb asymmetry is common in adolescents and may be a factor causing pain the spine and may also be associated with functional scoliosis of the lumbar region. Spine dysfunctions causing work related disabilities are highly prevalent throughout the world This public health issue is of multifactorial origin and affects he majority of those in mid life, the result of pelvic drop and increased lumbar lordosis. The aim of this work was to study the performance of our teenagers with respect to the mentioned variables. We analyzed 49 panoramic radiographs of the spines of schoolchildren between 13 and 17 years, 24 male and 25 female in the city of Temuco, Chile. Of total subjects 33 showed some degree of pelvic tilt which corresponds to $67.3 \%$ of subjects.. In 27 cases subjects were in the 1 to $10 \mathrm{~mm}$ range and 4 subjects in the 11 to $15 \mathrm{~mm}$ range. In 16 subjects no deviation was observed in frontal plane and 33 subjects had some degree of deviation. It is important to know these parameters in our adolescent population, to include formal education prevention in all aspects of life and to reduce the symptoms and costs of care and rehabilitation..

KEY WORDS: Pelvic; Pelvic asymmetry; Lumbar spine.

\section{REFERENCIAS BIBLIOGRAFICAS}

Anderson, J. A. Epidemiology aspects of back pain. J. Soc. Occup. Med., 36:90-4, 1986.

Balague, F.; Dutoit, G. \& Waldurger, M. Low back pain in school children. Scand. J. Rehabil. Med., 20:175-9, 1988.

Beal, M. C. Review of the short leg problem. J. Am. Osteopath. Assoc., 50:109-21, 1950.

Blake, R. L. \& Ferguson H. Limb length discrepancies. J. Am. Podiatr. Assoc., 82:33-8, 1992.

Carey, T.; Evans, A.; Hadler, N.; Kalsbeek, W.; McLaughlin, C. \& Fryer, J. Care-seeking among individuals with chronic low back pain. Spine, 20:312-7, 1995.

Centers for Disease Control and Prevention (CDC). Prevalence of disabilities and associated health conditions among adults. United States, 1999. JAMA, 285:1571-2, 2001.

Clark, G. R. Unequal leg length: an accurate method of detection and some clinical results. Rheumatol. Phys. Med., 11:385-90, 1972.
Cuccia, A. \& Caradonna, C. The relationship between the stomatognathic system and body posture. Clinics, 64:61-6, 2009.

Cummings, G.; Scholz, J. P. \& Barnes, K. The effect of imposed leg length difference on pelvic bone symmetry. Spine, 18:36873, 1993.

Del Sol, M.; \& Hunter, K. Evaluación postural de individuos mapuches de la zona costera de la IX Región de Chile. Int. J. Morphol., 22:339-42, 2004.

Dickson, R.; Stamper, P.; Sharp, A.; \& Harker, P. School screening for scoliosis: cohort study of clinical course. Br. Med. J., 281:65-7, 1980.

Espinoza-Navarro, O.; Valle, S.; Berríos, G.; Horta, J.; Rodríguez, H.; Rodríguez, M. Prevalencia de alteraciones posturales en niños de Arica-Chile. Efectos de un programa de mejoramiento de la postura. Int. J. Morphol., .27:25-30, 2009.

Fann, A.; Lee, R. \& Verbois, G. The Reliability of Postural XRays in Measuring Pelvic Obliquity. Arch. Phys. Med. Rehabi., 80:458-61, 1999. 
Florenciano, J. \& Gironés, P. Dismetría en extremidades inferiores y equilibrio de la pelvis. El Peu, 29(4):196-202, 2009.

Friberg, O.; Koivisto, E. \& Wegelius, C. Radiographic method for measurement of leg length inequality. Diagn. Imag. Clin. Med., $54: 78-81,1985$.

Frimoyer, J.; Pope, M.; Clements, J. H.; Wilder, D. G.; MacPherson, B. \& Ashikaga, T. Risk factors in low back pain. An epidemiologic survey. J. Bone Joint Surg., 65:213-8, 1983.

Fuentes, R.; Freesmeyer, W. \& Henríquez, J. Influencia de la postura corporal en la prevalencia de las disfunciones craneomandibulares. Rev. Med. Chile, 127:1079-85, 1999.

Gofton, J. \& Trueman, G. Studies in osteoarthritis of the hip: part II. osteoarthritis of the hip and leg-length disparity. Can. Med. Assoc. J., 104:791-9, 1971.

Gross, M.; Burns, C.; Chapman, S.; Hudson, C.; Curtis, H. Lehmann, J. \& Renner, J. Reliability and validity of rigid lift and pelvic leveling device method in assessing functional leg length inequality. J. Orthop. Sports Phys. Ther., 27:285-94, 1998.

Irvin, R. E. Reduction of lumbar scoliosis by use of a heel lift to level the sacral base. J. Am. Osteopath. Assoc., 91:37-44, 1991.

Krawiec, C.; Denegar, C.; Hertel, J.; Salvaterra, G. \& Buckley, W. Static innominate asymmetry and leg length discrepancy in asymptomatic collegiate athletes. Man. Ther., 8:207-13, 2003.

Levangie, P. The association between static pelvic asymmetry and low back pain. Spine, 24:1234-42, 1999.

Mackenzie, R. The lumbar spine. 15 Reprinted. Spinal publications New Zealand, New Zealand, 1998.

McKinnon, M.; Vickers, M. Ruddock, V. Townsend, J. \& Meade, T. Community studies of the health services implications of low back pain. Spine, 22:2161-6, 1997.

Morote, J. Estudio clínico y etiologico de la desigualdad en longitudes de los miembros inferiores. R. Esp. Cir. Ost., 11:255-65, 1976.

Okun, S.; Morgan, J. \& Burns, M. Limb length discrepancy: a new method of measurement and its clinical significance. J. Am. Podiatr. Assoc., 72:595-9, 1982.

Penha, P.; Amado, S.; Casarotto, A.; Amino, C. \& Caielli, D. Postural Assessment of girls between 7 and 10 years of age. Clinics, 60:9-16, 2005.

Silva, H. Ángulo del plano sagital de la columna lumbosacra en una muestra de adolescente de la ciudad de Temuco-Chile. Rev. Chil. Anat., 19:271-7, 2001.
Stewart, W.; Ricci, J.; Chee, E.; Morganstein, D. \& Lipton, R. Lost productive time and cost due to common pain conditions in the US workforce. JAMA, 290:2443-54, 2003.

Tilley, P. Radiographic identification of the sacral base. J. Am. Osteopath. Assoc., 65:1177-83, 1966.

Timgren, J. \& Soinila, S. Reversible pelvic asymmetry an overlooked syndrome manifesting as scoliosis apparent leg length difference and neurologic symptoms. J. Manipulative Physiol. Ther., 29:561-5, 2006.

Vismara L, Menegoni F, Zaina F, Galli M, Negrini S, Capodaglio P. Effect of obesity and low back pain on spinal mobility: a cross sectional study in women. J. Neuroeng. Rehabil., 7:3, 2010 .

Dirección para correspondencia:

Prof. Mg. Héctor Silva Mella

Depto. Ciencias Básicas

Facultad de Medicina

Universidad de La Frontera

Casilla 54-D, Temuco

CHILE

Email:hsilva@ufro.cl

Recibido : 03-10-2011

Aceptado: 02-11-2011 